\title{
A Case of Pulmonary Mycetoma Caused by Paecilomyces variotii
}

\author{
Diogo Paixão Marques ${ }^{1}$, Joana Carvalho ${ }^{2}$, Sara Rocha ${ }^{1}$, Raquel Domingos ${ }^{1}$ \\ ${ }^{1}$ Internal Medicine Department, Hospital de Egas Moniz, Lisbon, Portugal \\ ${ }^{2}$ Pulmonology Department, Hospital de Egas Moniz, Lisbon, Portugal
}

How to cite this article: Paixão Marques D, Carvalho J, Rocha S, Domingos R. A case of pulmonary mycetoma caused by Paecilomyces variotii. EJCRIM 2019;6: doi:10.12890/2019_001040.

Conflicts of Interests: The Authors declare that there are no competing interests.

This article is licensed under a Commons Attribution Non-Commercial 4.0 License

\section{ABSTRACT}

Fungal infections caused by Paecilomyces species are rare. Although most cases of human infection occur in immunocompromised patients, this species is also being increasingly recognized as a pathogen in immunocompetent individuals. Paecilomyces variotii and $P$. lilacinus are the most common pathogens in humans. P. variotii infection usually manifests as peritonitis, pneumonia or endophthalmitis. We present the case of an 84-year-old immunocompromised patient with interstitial lung disease, who developed pulmonary mycetoma with isolation of $P$. variotii in bronchoalveolar lavage fluid culture. We found one similar case in the literature, but Paecilomyces species was not identified. Therefore, this is the first described case of pulmonary mycetoma caused by P. variotii.

\section{LEARNING POINTS}

- Paecilomyces are rare causes of fungal infection in humans and occur more frequently in immunocompromised patients.

- Paecilomyces variotii is one of the most common pathogens of this species and is associated with peritonitis, pneumonia, osteomyelitis, endophthalmitis and fungaemia.

- To the date, pulmonary mycetoma caused by P. variotii has been described in only one patient in the literature.

\section{KEYWORDS}

Paecilomyces variotii, mycetoma, immunosuppression, interstitial lung disease

\section{INTRODUCTION}

Paecilomyces is a ubiquitous worldwide fungus that lives in the soil, decaying plants, wood and some food products ${ }^{[1]}$. These filamentous fungi are able to grow under stressful conditions such as hot or dry environments, which may contribute to their potential pathogenic nature ${ }^{[2]}$. Fungal infections caused by Paecilomyces species are uncommon ${ }^{[3]}$. Most cases of human infection occur in immunocompromised individuals, as well as following trauma or surgical procedures such as intraocular lens implantation ${ }^{[3]}$. However, Paecilomyces infection in immunocompetent patients have been increasingly reported in the literature ${ }^{[3,4]}$.

We present the case of an immunocompromised 84-year-old man with interstitial lung disease who developed pulmonary mycetoma caused by Paecilomyces variotii. 


\section{CASE DESCRIPTION}

An 84-year-old man presented to the Emergency Department complaining of productive cough and low-grade fever for the last few days. His past medical history included arterial hypertension, atrial fibrillation, ischaemic heart disease and nummular eczema. Nine years before admission he had presented to the pulmonology outpatient clinic with a few months' history of dry cough, fatigue and exertional dyspnoea. A chest CT scan had revealed subpleural bibasilar interlobular septal thickening (Fig. 1).
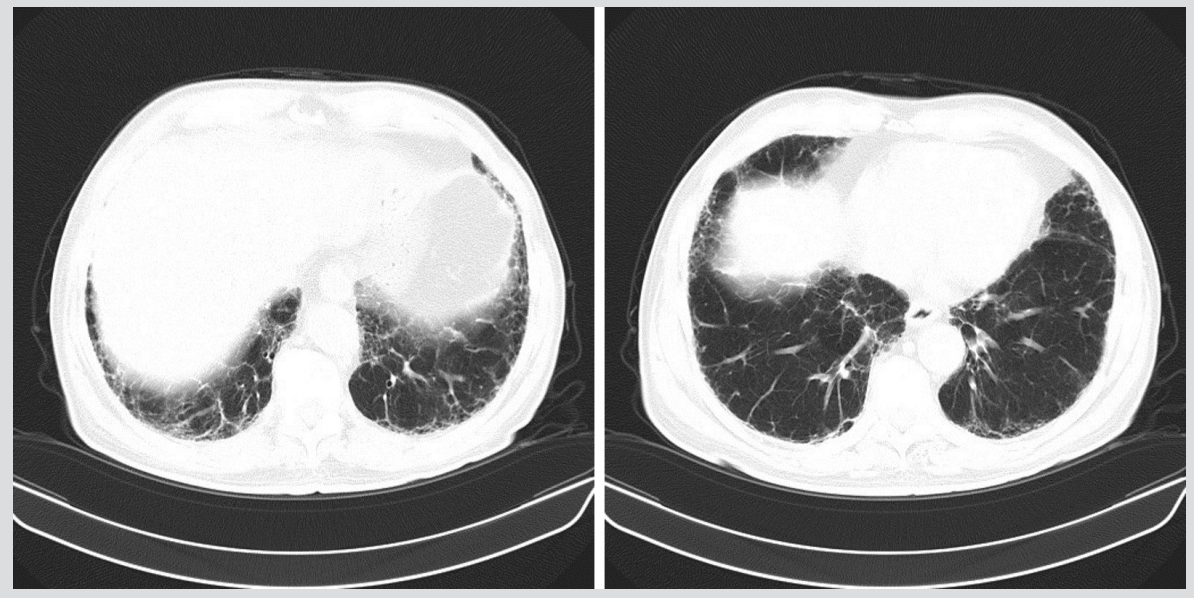

Figure 1. Initial chest CT scan revealing interlobular and intralobular septal thickening predominantly in a basal subpleural location

Bronchoalveolar lavage fluid showed 42\% lymphocytes with a diminished CD4/CD8 ratio. He had a history of previous exposure to birds but denied any other occupational or recreational exposures. A lung biopsy was not performed because of the high surgical risk. A diagnosis of probable chronic hypersensitivity pneumonitis-bird fancier's lung-was established and he was treated with corticosteroids but subsequently failed to completely avoid contact with birds. Despite maintaining treatment with prednisone $7.5 \mathrm{mg} / \mathrm{day}$ because of his nummular eczema, over the following years he showed steady clinical and radiological deterioration.

At presentation in the Emergency Department, he was febrile $\left(38.2^{\circ} \mathrm{C}\right)$, with tachypnoea at rest and a peripheral oxygen saturation at room air of $81 \%$; remaining vital signs were stable. Lung auscultation revealed bilateral crackles in lower lobes. There were no other relevant physical findings. Laboratory evaluation showed raised inflammatory markers (leucocyte count 19,400/ul with an absolute neutrophil count

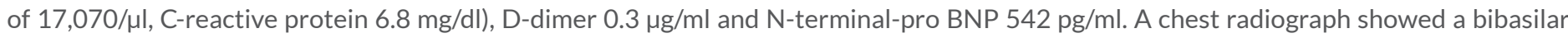
reticular pattern without parenchymal consolidation or pleural effusion (Fig. 2). A diagnosis of acute tracheobronchitis was made and antibiotic therapy with levofloxacin was started. The patient was admitted to the Internal Medicine Department.

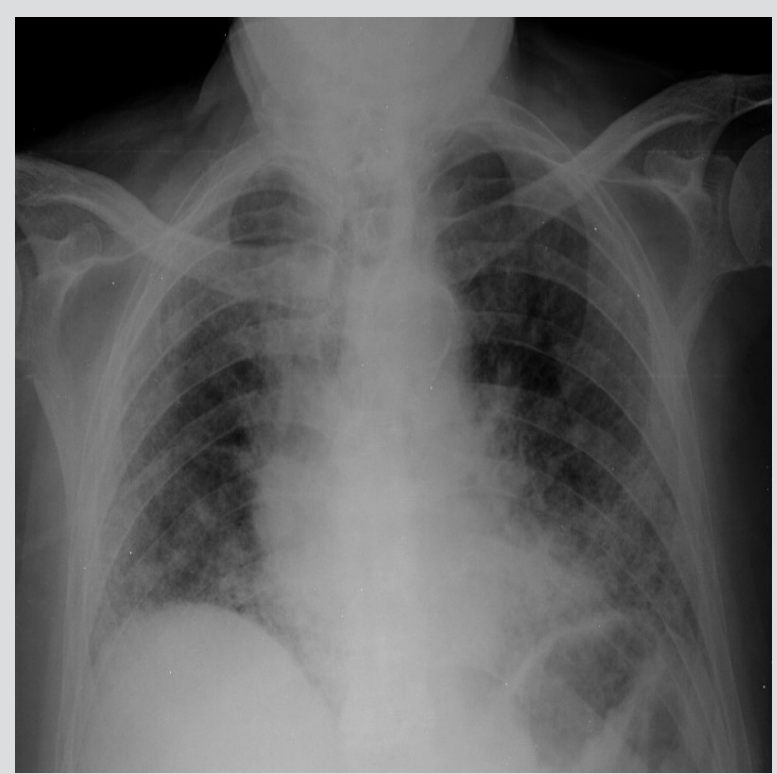

Figure 2. Chest radiograph showing a bilateral reticular pattern predominantly involving the lower lung zones and subpleural lung regions, suggestive of extensive fibrosis 
During the patient's hospital stay there was clinical improvement upon antibiotic therapy. A follow-up chest CT scan revealed a reticular pattern with traction bronchiectasis, honeycombing and multiple cystic lesions with a subpleural and basal predominance, some of which had a crescent-air sign, suggestive of mycetoma (Fig. 3).
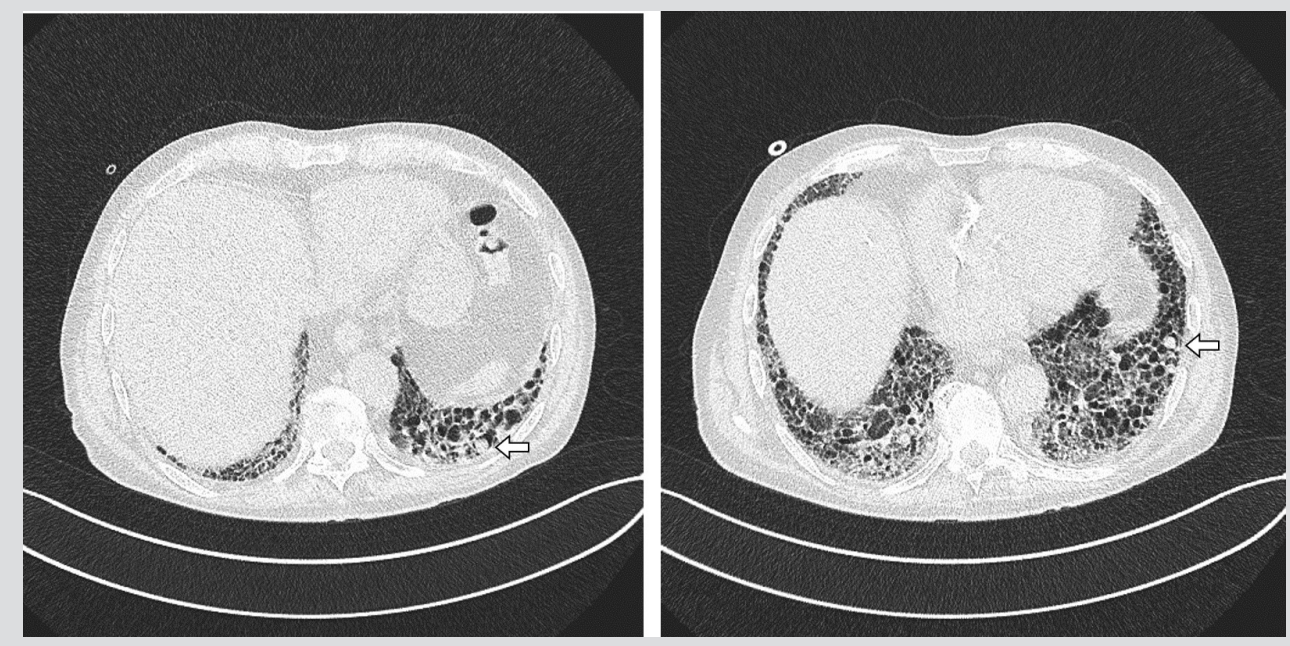

Figure 3. Follow up chest CT scan showing extensive lung fibrosis with multiple cystic lesions, some of which have a typical air-crescent sign suggestive of mycetoma (arrow)

A bronchoscopy was performed and the bronchoalveolar lavage fluid culture isolated $P$. variotii. Antifungal susceptibility testing showed a low minimal inhibitory concentration (MIC) to posaconazole and amphotericin B $(0.02 \mu \mathrm{g} / \mathrm{ml}$ and $0.06 \mu \mathrm{g} / \mathrm{ml}$, respectively) and a high MIC to voriconazole $(>32 \mu \mathrm{g} / \mathrm{ml})$. Serum and bronchoalveolar lavage galactomannan antigen detection was negative, as well as routine blood and sputum cultures. Given the context of immunosuppression and interstitial lung disease, a 6-week course of antifungal therapy with posaconazole was started. The patient was discharged after 7 days of antibiotic therapy.

Six days after hospital discharge, the patient was readmitted with nosocomial pneumonia. He was treated empirically with piperacillintazobactam and linezolid and maintained posaconazole. Nevertheless, he developed worsening respiratory failure and was later transferred to the intensive care unit where high-flow oxygen therapy was instituted. The patient refused mechanical ventilation. Despite completing a 14-day course of antibiotics he showed no clinical improvement, with progressive worsening of dyspnoea and respiratory failure eventually leading to death.

\section{DISCUSSION}

$P$. variotii and P. lilacinus are the most common pathogens of the Paecilomyces species ${ }^{[1,3,5]}$. Clinical manifestations of $P$. variotii infection include peritonitis, pneumonia, osteomyelitis, skin and soft tissue infections and fungaemia. $P$. variotii pneumonia is rare, with only a few cases reported in the literature ${ }^{[1]}$. Symptoms usually include productive cough, pleuritic chest pain and fever, which are mostly indistinguishable from those of pneumonia due to other bacterial or fungal causes ${ }^{[1]}$. Chest imaging abnormalities include hilar lymphadenopathy, confluent and patchy opacities and cavities ${ }^{[1]}$. The patient described in the present case did not seem to fall into this category since he showed clinical improvement with antibiotic therapy and the cystic lesions described in the chest CT scan were likely to secondary to progressive parenchymal fibrosis with architectural distortion.

The CT scan performed during the patient's hospital stay showed an air-crescent sign in some cystic lesions, suggestive of pulmonary mycetoma. Most pulmonary mycetomas are caused by Aspergillus, although Zygomycetes and Fusarium species have also been reported ${ }^{[3]}$. To our knowledge, only one case has been described in the literature of pulmonary mycetoma caused by Paecilomyces species ${ }^{[3]}$. Gutiérrez et al. described a case in which Paecilomyces was isolated by DNA sequencing techniques from a transthoracic needle aspiration culture of a large pulmonary mycetoma in an immunocompetent patient ${ }^{[3]}$. However, the clinical strain was not identified. Therefore, to our knowledge, the present case is the only example of $P$. variotii pulmonary mycetoma in a patient with interstitial lung disease.

Unlike $P$. lilacinus, $P$. variotii is usually susceptible to most antifungal agents apart from voriconazole and ravuconazole ${ }^{[1,4,5]}$. Therefore, posaconazole or amphotericin B are usually good alternatives to treat this infection ${ }^{[4,5]}$. Correct species identification and antifungal susceptibility testing are crucial to guide appropriate therapy. Since treatment guidelines are lacking, duration of treatment must be tailored according to clinical response. 


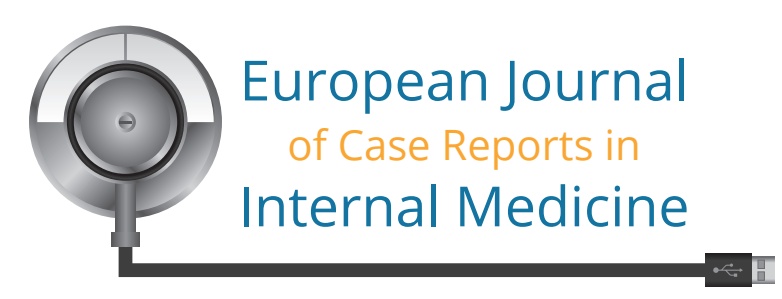

Most cases of pulmonary aspergilloma require surgery; however, surgery was not a suitable option for this patient due to the high surgical risk and multifocal distribution of mycetomas. Unfortunately, this patient died even though he was treated with appropriate antifungal and antibacterial therapy. This was probably due to the severity of his underlying conditions, particularly the lung disease.

In conclusion, $P$. variotii is a rare but increasingly common cause of infection in both immunocompromised and immunocompetent individuals. Although it is very rare, this case shows that Paecilomyces species can also be aetiological agents of pulmonary mycetoma.

\section{REFERENCES}

1. Steiner B, Aquino VR, Paz AA, Silla LM, Zavascki A, Goldani LZ. Paecilomyces variotii as an emergent pathogenic agent of pneumonia. Case Rep Infect Dis 2013;2013:273848.

2. Houbraken J, Verweij PE, Rijs AJ, Borman AM, Samson RA. Identification of Paecilomyces variotii in clinical samples and settings. J Clin Microbiol 2010;48:2754-2761.

3. Gutiérrez F, Masiá M, Ramos J, Elía M, Mellado E, Cuenca-Estrella M. Pulmonary mycetoma caused by an atypical isolate of Paecilomyces species in an immunocompetent individual: case report and literature review of Paecilomyces lung infections. Eur J Clin Microbiol Infect Dis 2005;24:607-611.

4. Feldman R, Cockerham L, Buchan BW, Lu Z, Huang AM. Treatment of Paecilomyces variotii pneumonia with posaconazole: case report and literature review. Mycoses 2016;59:746-750.

5. Aguilar C, Pujol I, Sala J, Guarro J. Antifungal susceptibilities of Paecilomyces species. Antimicrob Agents Chemother 1998;42:1601-1604. 